OPEN ACCESS

Edited by: Lucia Gardossi, University of Trieste, Italy

Reviewed by: Samarthya Bhagia, Oak Ridge National Laboratory,

United States

Madalina Tudorache, University of Bucharest, Romania

*Correspondence:

Li Shuai

lishuai@fafu.edu.cn

${ }^{\dagger}$ These authors have contributed equally to this work and share first authorship

Specialty section:

This article was submitted to Industrial Biotechnology,

a section of the journal Frontiers in Bioengineering and Biotechnology

Received: 21 September 2021 Accepted: 01 November 2021 Published: 18 November 2021

Citation: Yang G, Luo X and Shuai L (2021) Bioinspired Cellulase-Mimetic Solid Acid Catalysts for Cellulose Hydrolysis. Front. Bioeng. Biotechnol. 9:770027. doi: 10.3389/fbioe.2021.770027

\section{Bioinspired Cellulase-Mimetic Solid Acid Catalysts for Cellulose Hydrolysis}

\author{
Guangxu Yang ${ }^{\dagger}$, Xiaolin $\mathrm{Luo}^{\dagger}$ and Li Shuai * \\ College of Materials Engineering, Fujian Agriculture and Forestry University, Fuzhou, China
}

Glucose produced by catalytic hydrolysis of cellulose is an important platform molecule for producing a variety of potential biobased fuels and chemicals. Catalysts such as mineral acids and enzymes have been intensively studied for cellulose hydrolysis. However, mineral acids show serious limitations concerning equipment corrosion, wastewater treatment and recyclability while enzymes have the issues such as high cost and thermal stability. Alternatively, solid acid catalysts are receiving increasing attention due to their high potential to overcome the limitations caused by conventional mineral acid catalysts but the slow mass transfer between the solid acid catalysts and cellulose as well as the absence of ideal binding sites on the surface of the solid acid catalysts are the key barriers to efficient cellulose hydrolysis. To bridge the gap, bio-inspired or bio-mimetic solid acid catalysts bearing both catalytic and binding sites are considered futuristic materials that possess added advantages over conventional solid catalysts, given their better substrate adsorption, high-temperature stability and easy recyclability. In this review, cellulase-mimetic solid acid catalysts featuring intrinsic structural characteristics such as binding and catalytic domains of cellulase are reviewed. The mechanism of cellulasecatalyzed cellulose hydrolysis, design of cellulase-mimetic catalysts, and the issues related to these cellulase-mimetic catalysts are critically discussed. Some potential research directions for designing more efficient catalysts for cellulose hydrolysis are proposed. We expect that this review can provide insights into the design and preparation of efficient bioinspired cellulase-mimetic catalysts for cellulose hydrolysis.

Keywords: glucose, cellulase, solid acid, cellulase-mimetic catalysts, cellulose hydrolysis

\section{INTRODUCTION}

Glucose, resulting from hydrolysis of cellulose, is an important biomass-derived platform molecule for producing a variety of value-added fuels and chemicals (Yabushita et al., 2014). Many efforts are ongoing to reduce glucose production cost for economic biorefineries. To a large extent, the efficiency of catalysts for cellulose hydrolysis affects the economy of glucose production. A good catalyst should selectively and cost-effectively convert cellulose to glucose in high concentrations with limited glucose degradation.

Mineral acids and enzymes (such as cellulase) have been widely used as catalysts for cellulose hydrolysis. Despite the low cost of mineral acids, mineral acid-catalyzed cellulose hydrolysis has issues such as glucose degradation, equipment corrosion, and wastewater treatment (Palkovits et al., 2010; Orozco et al., 2011; Qiao et al., 2018; Zhou et al., 2021). In contrast, cellulase can selectively catalyze cellulose hydrolysis at mild conditions but the cost of cellulase loadings required for efficient conversion of cellulosic materials to glucose accounts for a large portion of the whole processing cost. Moreover, cellulase has the highest catalytic activity only under an optimum condition; a varied 

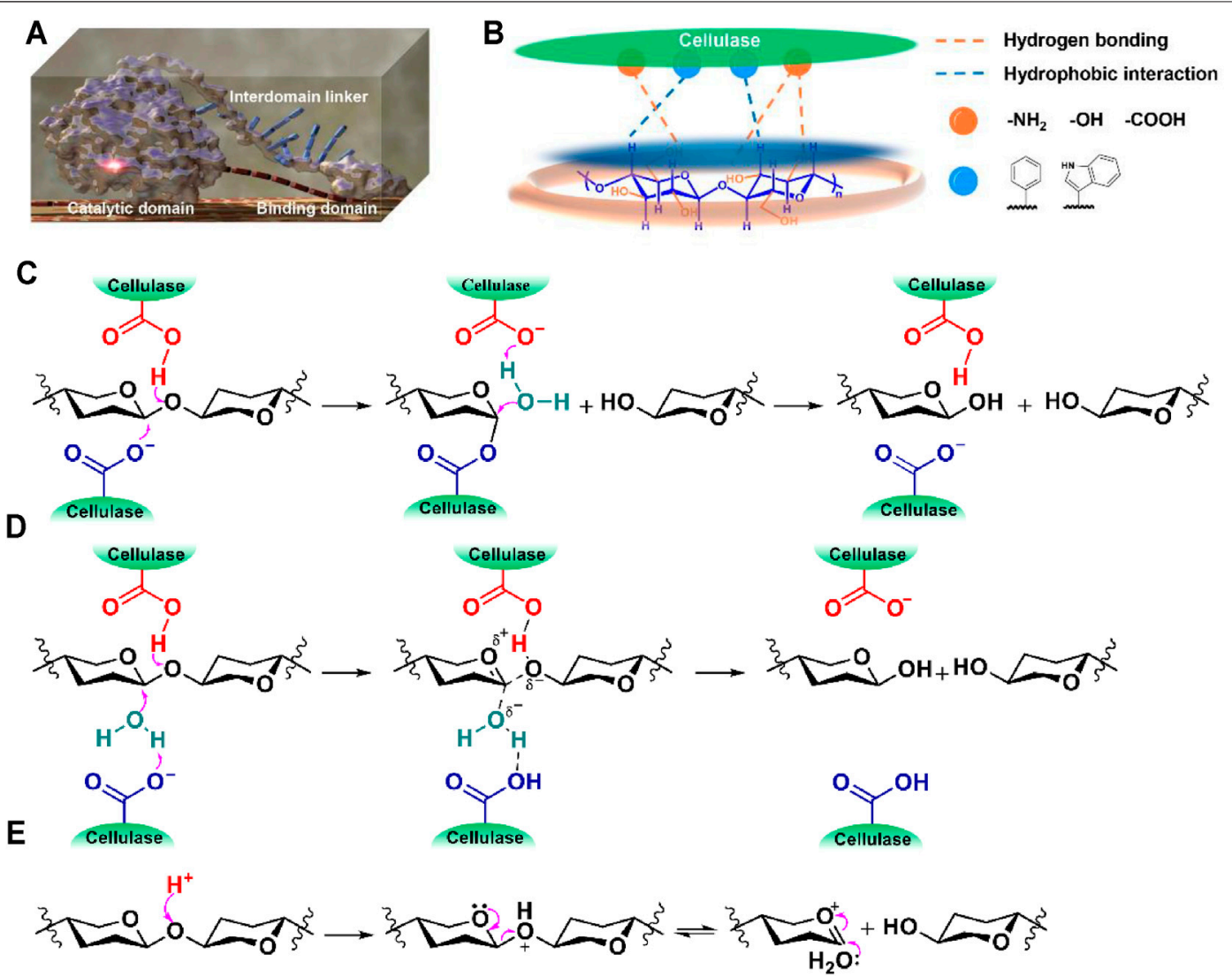

FIGURE 1 | (A) A modeling image of cellulase, (B) cellulase-cellulose binding via hydrogen bonding (orange) and hydrophobic interactions (blue), catalytic hydrolysis of cellulose by cellulase in (C) a retaining or (D) an inverting mechanism, and (E) a proposed mechanism of mineral acid-catalyzed cellulose hydrolysis (substituted groups are omitted for better visibility).

condition (e.g. a higher or lower temperature or $\mathrm{pH}$ value) inhibits cellulase activity and even denatures cellulase; therefore, the rate of enzymatic hydrolysis cannot be accelerated through an increase in the reaction temperature (Berlin et al., 2007; dos Santos et al., 2018; Huang, 1975). In consideration of recyclability and stability, solid acid catalysts have been widely explored in recent years while the efficiency of cellulose hydrolysis catalyzed by these catalysts is highly restricted by the limited interactions between the catalysts and cellulose (Hara, 2010; Shimizu et al., 2011; Huang et al., 2013; Hu et al., 2015; Wang et al., 2015) To overcome this limitation, novel cellulase-mimetic catalysts with both cellulose-binding and catalytic sites were developed in the last decade (Shuai et al., 2012). The catalytic sites consisting of acid-base pairs can stabilize the intermediate product (i.e., an oxocarbenium ion) during the cleavage of the glycosidic bond, thereby reducing the energy barrier of cellulose hydrolysis; the binding group on the surface of a cellulase-mimetic catalyst can associate the catalyst with cellulosic materials, promoting the mass transfer rate between the catalyst and cellulose in such a heterogeneous reaction.

In this review, we summarize the recent studies regarding cellulase-mimetic catalysts. In particular, the mechanism of cellulase-catalyzed cellulose hydrolysis and the structural characteristics of cellulase-mimetic catalysts such as binding and catalytic sites are critically reviewed. In the end, we discuss the issues of the current catalyst design and propose some potential directions for synthesizing more efficient cellulase-mimetic catalysts.

\section{CELLULASE-CATALYZED CELLULOSE HYDROLYSIS}

Structurally, cellulase comprises at least three separate structural elements of different functions, i.e., a catalytic domain (CD), a cellulose-binding domain (CBD), and an interdomain linker (Figure 1A; Rabinovich et al., 2002). The CBD is responsible for associating cellulase with cellulose while the $\mathrm{CD}$ catalyzes the cleavage of glycosidic bonds of a cellulose chain. During the enzymatic hydrolysis of cellulose, cellulase is adsorbed onto the bulk solid cellulosic materials through hydrophobic interactions (e.g., $\mathrm{CH}-\pi$ interaction) between the $\mathrm{CBD}$ containing the aromatic and alkyl residues of amino acids and the axial face of cellulose as well as hydrogen bonding interactions between the 
polar groups of amino acids and cellulose hydroxyls (Figure 1B; Asensio et al., 2012; Georgelis et al., 2012), then the cleavage of the glycosidic bond of the captured cellulose chain is catalyzed by a catalytic site in a retaining or inverting mechanism (Figures 1C,D; Badieyan et al., 2012; Zechel et al., 2000). In both of the proposed hydrolysis mechanisms, a carboxylic acid (COOH)carboxylate $\left(\mathrm{COO}^{-}\right)$pair (an acid-base pair) works synergistically to cleave the glycosidic bond (Figures 1C,D). The nucleophilic attack of $\mathrm{COO}^{-}$to the anomeric carbon of cellulose stabilizes the high-energy free oxocarbenium ions that is usually formed in an acid-catalyzed hydrolysis process (Figure 1E; Brown et al., 1979; Rinaldi et al., 2010), thereby reducing the energy barrier for cellulose hydrolysis. During the enzymatic hydrolysis of cellulose, the occurrence of such an enzymatic reaction highly depends on the perfect conformation of the acid-base pair. When the glycosidic bond of a cellulose chain is perfectly fit into the acid-base pairing site, the energy barrier for cellulose hydrolysis is substantially lowered. Such perfect fitting is assisted by the CBD which associates cellulase with cellulose chains via specific adsorption. The adsorption of cellulase to cellulose increases the mass transfer rate between them, thereby further increasing the enzymatic hydrolysis efficiency.

A temperature or $\mathrm{pH}$ change would substantially affect the structural conformations of the catalytic and binding sites and thereby the catalytic activity of cellulase. The hydrolysis rate of cellulose is thus strictly limited by the optimum temperature for cellulase and cannot be improved via changing the reaction temperature. In contrast, a temperature increase can enhance the rate of acid-catalyzed hydrolysis without changing the structure of chemically synthesized solid acid catalysts. Therefore, solid acid catalysts are receiving increasing attention due to its high potential to overcome the limitation of cellulase.

\section{CELLULASE-MIMETIC CATALYSTS}

As conventional solid acids do not have specific sites for binding cellulose and specific acid-base pairs for catalyzing cellulose hydrolysis, the rate of cellulose hydrolysis highly relies on the mass transfer rate between the acid catalysts and cellulose as well as the acidity of the catalysts.

Particularly, the mass transfer rate is highly restrained when a solid acid is used as a catalyst. An elevated temperature could enhance the mass transfer rate but it would cause more cellulose and glucose degradation and thereby low glucose selectivity (Zuo et al., 2014). Alternatively, synthesis of cellulase-mimetic solid acid containing both catalytic and cellulose-binding sites (groups) is a viable way to improve the cellulose hydrolysis efficiency.

\section{Mimetic Design of Catalytic Sites}

The mechanism of cellulase-catalyzed cellulose hydrolysis indicates the important role of the $\mathrm{COOH}-\mathrm{COO}^{-}$pair in the cleavage of the glycosidic bonds. Incorporation of $\mathrm{COOH}-\mathrm{COO}^{-}$ (Cho et al., 2015; Sugano et al., 2011) or similar acid-base pairs (e.g. $\mathrm{COOH}-\mathrm{NH}_{2}$ ) (Chen et al., 2019; Cho et al., 2016) onto catalyst supporting materials (e.g. carbon nanotubes (CNT)
(Sugano et al., 2011), and magnetic silica-based nanoparticles (Cho et al., 2015), and green magnetic nano-catalyst (GMN) (Cho et al., 2016) to mimic the catalytic conformation of cellulase was therefore explored extensively (Figure 2). Since the theoretical $\mathrm{pKa}$ value of the carboxylic group is around 3.1-4.4, the $\mathrm{pH}$ value of the hydrolysis reaction media is generally adjusted to around 3.0 to ensure the presence of the acid-base (or conjugated base) pair in a reaction medium for the best performance (Cho et al., 2015; Sugano et al., 2011). In contrast to the conventional acidcatalytic hydrolysis processes that require high temperatures, some of these catalysts (e.g., a green magnetic nano-catalyst (GMN) with carboxyl and imidazole groups as an acid-base pair) could catalyze the cleavage of the glycosidic bonds under a very mild condition $\left(37.5^{\circ} \mathrm{C}, \mathrm{pH}=5\right)$ in a similar manner to cellulase (Cho et al., 2016). However, these catalysts are far less active than cellulase because the random distribution of acid (such as - $\mathrm{COOH}$ and phenolic- $\mathrm{OH}$ ) and base (such as $\mathrm{COO}^{-}$and $\mathrm{NH}_{2}$ ) groups on the surface of the catalysts does not completely mimic the ideal structural conformation of the $\mathrm{COOH}-\mathrm{COO}^{-}$ pair in cellulase. To understand the effect of well-arranged acidbase pairs on the hydrolysis efficiency, a comparative study of ortho-, meta-, and para-hydroxybenzoic acids (pKa values of 3.0, 4.1 , and 4.6, respectively) as the catalysts for cellulose hydrolysis was conducted (Yabushita, 2016). The considerably higher catalytic activity of ortho-hydroxybenzoic acid (a turnover frequency (TOF) of $28 \mathrm{~h}^{-1}$ ) than others (TOFs of 5.7 and $2.4 \mathrm{~h}^{-1}$ for meta- and para-hydroxybenzoic acids, respectively) suggests the major contribution of a vicinal acid-base pair to the catalytic activity of a synthesized catalyst (Yabushita, 2016). The vicinal acid-base pair has a better conformation than others towards stabilizing the intermediate oxocarbenium ion and protonating the hydroxyl ion formed during the cleavage of the glycosidic bond. Consistently, a polymer acid catalyst, hyperbranched poly(arylene oxindole) (5-OH-SHPAO) functionalized with phenolic hydroxyls and ortho-substituted sulfonic acids as vicinal acid-base pairs demonstrated high catalytic activity for cellulose hydrolysis and achieved a cellulose conversion of $88 \%$ with a glucose yield of $56 \%$ and above $93 \%$ selectivity to all useful hydrolytic and dehydration products (such as lactic acid) at $170^{\circ} \mathrm{C}$ in $4 \mathrm{~h}$ (Figure 2; Yu et al., 2016). During the hydrolysis, the phenolic hydroxyl attacks the anomeric carbon to stabilize the oxocarbenium ion and the resulting hydroxyl ion can be readily protonated by the neighboring sulfonic acid. In contrast to the findings of Yabushita et al. where the strict requirement of a vicinal acidbase site is preferred for constructing the catalytic site (Yabushita, 2016), a biochar sulfonic acid bearing randomly distributed polyamide chains (BCSA-PA) also demonstrated higher activity for cellulose hydrolysis (26\% reducing sugars (RSs) and $23 \%$ HMF yields) than BCSA without PA (22\% RSs and $<0.1 \%$ HMF yields) (Figure 2; Chen et al., 2019). The finding indicates that the flexible polyamide chain can easily move to attack the anomeric carbon to stabilize the oxocarbenium ion, reducing the energy barrier of the cleavage of the glycosidic bonds.

In summary, the merit of constructing of a catalytic acid-base pair is to stabilize the intermediate and enable the occurrence of 


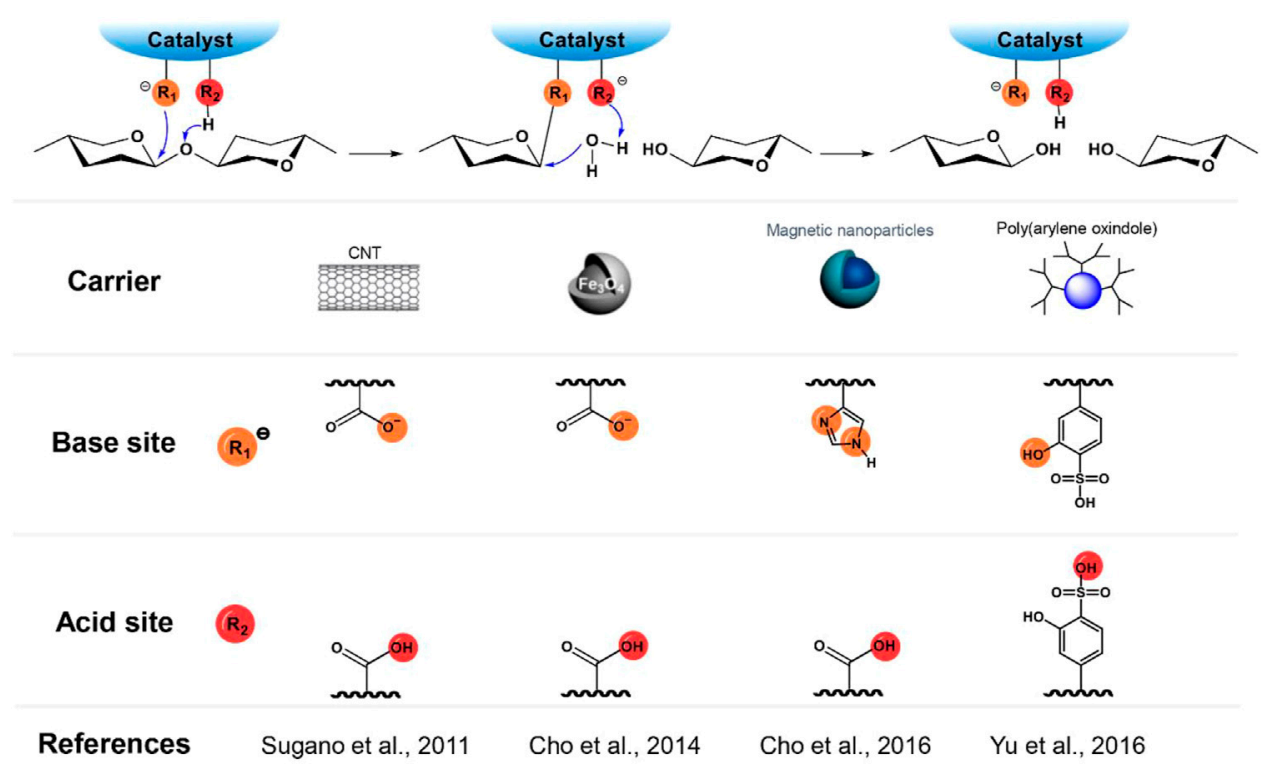

FIGURE 2 | A summary of acid-base pairs as catalytic sites of cellulose-mimetic solid acid catalysts and corresponding mechanism for the catalytic cleavage of glycosidic bonds in cellulose chain.

the hydrolysis under mild conditions and even without the use of strong acid catalysts. However, it is very challenging to design a solid acid that can completely mimic the naturally evolved catalytic conformation of cellulase. Due to such a challenge, current cellulase-mimetic solid acid catalysts are not comparable to cellulase in terms of the catalytic activity.

\section{Mimetic Design of Binding Sites}

The high efficiency of cellulase-catalyzed cellulose hydrolysis is due to not just the unique structural configuration of the catalytic site but also the assistance of the CBD in associating cellulase with cellulosic materials. In addition to the absence of ideal acid-base pairs, the slow mass transfer between solid acid catalysts and cellulose is another limitation to efficient hydrolysis. Incorporation of binding sites to a catalyst to enhance the mass transfer rate is therefore another important research area of cellulase-mimetic catalyst development. The catalytic performance of various cellulase-mimetic solid acid catalysts on hydrolyzing cellulose was compared in Table $\mathbf{1 .}$

\section{(A) Binding Via Hydrogen Bonding Interaction}

Initially, researchers found that carbon-based solid acids prepared from incomplete carbonization of natural organic matters followed by sulfonation exhibited remarkable hydrolysis performance with water-soluble glucose and glucan yields of 4-68\% (Suganuma et al., 2008; Toda et al., 2005). Further mechanistic studies revealed the contribution of the hydrogen bonding interaction between the phenolic $-\mathrm{OH}$ and $-\mathrm{COOH}$ on the catalyst surface and cellulose hydroxyls to the high catalytic activity of the carbon-based solid acids (Figure 3; Hu et al., 2015; Suganuma et al., 2008). The interaction renders enhanced mass transfer rates between the catalysts and cellulosic materials and thereby high hydrolysis efficiencies. This finding inspires studies on a variety of carbon-based catalysts prepared from different raw materials, such as mesoporous carbon (CMK-3) (Pang et al., 2010), mesoporous silicon oxide/carbon composite (Van de Vyver et al., 2010), magnetic oxide/sulfonated carbon shell composite (Zhang et al., 2013), graphene oxide (GO) (Kitano et al., 2009; Verma et al., 2013; Zhao et al., 2014; Mission et al., 2017; Zhang et al., 2017), and lignin (Hu et al., 2015; Zhu et al., 2016; Gan et al., 2017; Wang et al., 2020; Zhu et al., 2020). In these studies, GO-based catalysts demonstrate excellent performance for cellulose hydrolysis. The high surface area and abundant carboxylic and phenolic hydroxyl groups on the surface of the GO-based catalysts facilitate the formation of a considerable amount of hydrogen bonds with cellulose (Zhao et al., 2014). The apparent activation energy for cellulose hydrolysis catalyzed by a functionalized $\mathrm{GO} /$ iron nanoparticle hybrid material (FeGO-SO $\mathrm{S}_{3} \mathrm{H}$ ) was only $12 \mathrm{~kJ} \mathrm{~mol}^{-1}$, which is much lower than that for sulfuric acid-catalyzed hydrolysis under an optimal condition $\left(170 \mathrm{~kJ} \mathrm{~mol}^{-1}\right.$ ) (Verma et al., 2013). Recently, Yang et al. (Yang et al., 2021) also prepared porous polymeric solid acids (PPSAs) bearing hydroxyl and sulfonic acid groups for cellulose hydrolysis in water through the low-cost Friedel-Crafts "knitting" polymerization of hydroxyl-containing aromatic monomers followed by sulfonation. The reason for the high efficiency of the synthesized bifunctional catalysts (a glucose yield of $93 \%$ from Avicel at $120^{\circ} \mathrm{C}$ within $48 \mathrm{~h}$ ) was attributed to the porous structure and the presence of the hydroxyl (cellulose-binding group) on the solid acids.

The enhanced performance resulting from the hydrogen bonding interaction between carbon-based catalysts and cellulosic materials encouraged many attempts to examine other electronegative groups such as amine, and halide as 
TABLE 1 | The catalytic performance of various cellulase-mimetic solid acids catalysts on hydrolyzing cellulose.

\begin{tabular}{|c|c|c|c|c|c|c|c|c|c|c|}
\hline Entry & Catalyst & CBD & $\begin{array}{c}\text { Binding } \\
\text { interaction }\end{array}$ & Substrates & $\begin{array}{l}\text { Reaction } \\
\text { condition }\end{array}$ & $\begin{array}{c}\text { Conv. } \\
\text { (\%) }\end{array}$ & $\begin{array}{l}\text { Glu. } \\
\text { (\%) }\end{array}$ & $\begin{array}{c}\text { Other } \\
\text { products } \\
(\%)\end{array}$ & $\begin{array}{l}\text { Catalyst } \\
\text { stability }\end{array}$ & Ref \\
\hline 1 & $\mathrm{Fe}-\mathrm{GO}-\mathrm{SO}_{3} \mathrm{H}$ & $\begin{array}{l}-\mathrm{OH} \\
-\mathrm{COOH}\end{array}$ & $\begin{array}{l}\text { Hydrogen } \\
\text { bonding }\end{array}$ & Cellulose & $\begin{array}{c}\mathrm{H}_{2} \mathrm{O} \\
75^{\circ} \mathrm{C}, 44 \mathrm{~h}\end{array}$ & 95 & 50 & $\begin{array}{l}\text { Cellobiose (4.3) } \\
\text { 4-5unit } \\
\text { oligomeric (40) }\end{array}$ & $\begin{array}{l}\text { Kept stable after } \\
\text { recycling five times }\end{array}$ & $\begin{array}{l}\text { Verma } \\
\text { et al. } \\
\text { (2013) }\end{array}$ \\
\hline 2 & PPSAs & $-\mathrm{OH}$ & $\begin{array}{l}\text { Hydrogen } \\
\text { bonding }\end{array}$ & Avicel & $\begin{array}{c}\mathrm{H}_{2} \mathrm{O} \\
120^{\circ} \mathrm{C}, 48 \mathrm{~h}\end{array}$ & - & 93 & - & $\begin{array}{l}\text { From } 92.6 \% \text { to } \\
65.1 \% \text { after four } \\
\text { cycles }\end{array}$ & $\begin{array}{l}\text { Yang } \\
\text { et al. } \\
(2021)\end{array}$ \\
\hline 3 & $\mathrm{CP}-\mathrm{SO}_{3} \mathrm{H}$ & $-\mathrm{Cl}$ & $\begin{array}{l}\text { Hydrogen } \\
\text { bonding }\end{array}$ & Avicel & $\begin{array}{c}\mathrm{H}_{2} \mathrm{O} \\
120^{\circ} \mathrm{C}, 10 \mathrm{~h}\end{array}$ & - & 93 & - & $\begin{array}{l}\text { The activity did not } \\
\text { decline after } \\
\text { recycling three } \\
\text { times }\end{array}$ & $\begin{array}{l}\text { Shuai } \\
\text { et al. } \\
(2012)\end{array}$ \\
\hline 4 & $\begin{array}{l}\text { PTA@MIL- } \\
101-\mathrm{NO}_{2}\end{array}$ & $-\mathrm{NO}_{2}$ & $\begin{array}{l}\text { Hydrogen } \\
\text { bonding }\end{array}$ & Avicel & $\begin{array}{c}\mathrm{H}_{2} \mathrm{O}, 180^{\circ} \mathrm{C} \\
3-11 \mathrm{~h}\end{array}$ & - & 16.2 & - & $\begin{array}{l}\text { Decreased by } 4.3 \% \\
\text { after three times }\end{array}$ & $\begin{array}{l}\text { Han et al. } \\
\text { (2019) }\end{array}$ \\
\hline 5 & $\begin{array}{l}\text { PTA@MIL- } \\
\text { 101-Br }\end{array}$ & $-\mathrm{Br}$ & $\begin{array}{l}\text { Hydrogen } \\
\text { bonding }\end{array}$ & Avicel & $\begin{array}{c}\mathrm{H}_{2} \mathrm{O}, 180^{\circ} \mathrm{C} \\
3-11 \mathrm{~h}\end{array}$ & - & 10.1 & - & $\begin{array}{l}\text { Decreased by } 3.7 \% \\
\text { after three times }\end{array}$ & $\begin{array}{l}\text { Han et al. } \\
\text { (2019) }\end{array}$ \\
\hline 6 & $\begin{array}{l}\text { PTA@MIL- } \\
101-\mathrm{NH}_{2}\end{array}$ & $-\mathrm{NH}_{2}$ & $\begin{array}{l}\text { Hydrogen } \\
\text { bonding }\end{array}$ & Avicel & $\begin{array}{c}\mathrm{H}_{2} \mathrm{O}, 180^{\circ} \mathrm{C} \\
3-11 \mathrm{~h}\end{array}$ & - & 12.8 & - & $\begin{array}{l}\text { Kept stable after } \\
\text { recycling three } \\
\text { times }\end{array}$ & $\begin{array}{l}\text { Han et al. } \\
\text { (2019) }\end{array}$ \\
\hline 7 & $\begin{array}{l}\text { PTA@MIL- } \\
101-\mathrm{Cl}\end{array}$ & $-\mathrm{Cl}$ & $\begin{array}{l}\text { Hydrogen } \\
\text { bonding }\end{array}$ & Avicel & $\begin{array}{c}\mathrm{H}_{2} \mathrm{O}, 180^{\circ} \mathrm{C} \\
3-11 \mathrm{~h}\end{array}$ & - & 15.0 & - & $\begin{array}{l}\text { Decreased by } 3.9 \% \\
\text { after three times }\end{array}$ & $\begin{array}{l}\text { Han et al. } \\
(2019)\end{array}$ \\
\hline 8 & $\mathrm{CP}-\mathrm{SO}_{3} \mathrm{H}-1.69$ & $-\mathrm{Cl}$ & $\begin{array}{l}\text { Hydrogen } \\
\text { bonding }\end{array}$ & Avicel & $\begin{array}{c}\mathrm{H}_{2} \mathrm{O} \\
170^{\circ} \mathrm{C}, 10 \mathrm{~h}\end{array}$ & 100 & 2.1 & LA (33.1) & $\begin{array}{l}\text { Deactivated in two } \\
\text { runs }\end{array}$ & $\begin{array}{l}\text { Zuo et al. } \\
\text { (2014) }\end{array}$ \\
\hline 9 & SUCRA-SO ${ }_{3} \mathrm{H}$ & $-\mathrm{Cl}$ & $\begin{array}{l}\text { Hydrogen } \\
\text { bonding }\end{array}$ & $\begin{array}{l}\text { Hardwood (rice } \\
\text { straw) pretreated } \\
\text { by the ionic liquid }\end{array}$ & $\begin{array}{c}\mathrm{H}_{2} \mathrm{O} \\
120^{\circ} \mathrm{C}, 24 \mathrm{~h}\end{array}$ & - & $\begin{array}{c}12.7 \\
(19.5)\end{array}$ & $\begin{array}{l}\text { Xylose } \\
(44.4,57.3)\end{array}$ & $\begin{array}{l}\text { Remained around } \\
56 \% \text { after seven } \\
\text { times }\end{array}$ & $\begin{array}{l}\text { Hu et al. } \\
\text { (2014) }\end{array}$ \\
\hline 10 & $\mathrm{HA}-\mathrm{CC}-\mathrm{SO}_{3} \mathrm{H}$ & $-\mathrm{Cl}$ & $\begin{array}{l}\text { Hydrogen } \\
\text { bonding }\end{array}$ & Avicel & $\begin{array}{c}\mathrm{H}_{2} \mathrm{O} \\
155^{\circ} \mathrm{C}, 4 \mathrm{~h}\end{array}$ & 11.3 & 10.8 & - & $\begin{array}{l}\text { Kept stable after } \\
\text { recycling three } \\
\text { times }\end{array}$ & $\begin{array}{l}\text { Pang } \\
\text { et al. } \\
\text { (2014) }\end{array}$ \\
\hline 11 & $\mathrm{SA}-\mathrm{TsOH}$ & $-\mathrm{Cl}$ & $\begin{array}{l}\text { Hydrogen } \\
\text { bonding }\end{array}$ & Avicel & $\begin{array}{l}\text { [BMIM][Cl], } \\
130^{\circ} \mathrm{C}, 1 \mathrm{~h}\end{array}$ & - & - & TRS (67.6) & $\begin{array}{l}\text { From } 67.6 \text { to } 60.4 \% \\
\text { after eight cycles }\end{array}$ & $\begin{array}{l}\text { Shen } \\
\text { et al. } \\
\text { (2018) }\end{array}$ \\
\hline 12 & Solid acid & $-\mathrm{Cl}$ & $\begin{array}{l}\text { Hydrogen } \\
\text { bonding }\end{array}$ & Ball-milled Avicel & $\begin{array}{c}\mathrm{H}_{2} \mathrm{O} \\
120^{\circ} \mathrm{C}, 24 \mathrm{~h}\end{array}$ & - & 84.9 & - & $\begin{array}{l}\text { Lost activity after } \\
\text { four runs }\end{array}$ & $\begin{array}{l}\text { Yang } \\
\text { et al. } \\
(2016)\end{array}$ \\
\hline 13 & $\begin{array}{l}\mathrm{Fe}_{3} \mathrm{O}_{4} / \mathrm{Cl}- \\
\mathrm{MCMB}-\mathrm{SO}_{3} \mathrm{H}\end{array}$ & $-\mathrm{Cl}$ & $\begin{array}{l}\text { Hydrogen } \\
\text { bonding }\end{array}$ & Cellulose & $\begin{array}{c}\mathrm{H}_{2} \mathrm{O} \\
140^{\circ} \mathrm{C}, 3 \mathrm{~h}\end{array}$ & - & - & TRS (68.6) & $\begin{array}{l}\text { From } 68.6 \text { to } 61.1 \% \\
\text { after six cycles }\end{array}$ & $\begin{array}{l}\text { Li et al. } \\
(2020)\end{array}$ \\
\hline 14 & $\mathrm{Cl}-\mathrm{MCMB}-\mathrm{SO}_{3} \mathrm{H}$ & $-\mathrm{Cl}$ & $\begin{array}{l}\text { Hydrogen } \\
\text { bonding }\end{array}$ & Pretreated MCC & $\begin{array}{c}\mathrm{H}_{2} \mathrm{O} \\
130^{\circ} \mathrm{C}, 3 \mathrm{~h}\end{array}$ & - & - & TRS (70.3) & $\begin{array}{l}\text { Decreased by } 9.6 \% \\
\text { after five times }\end{array}$ & $\begin{array}{l}\text { Li et al. } \\
(2020)\end{array}$ \\
\hline 15 & $\mathrm{CMC}-\mathrm{SO}_{3} \mathrm{H}$ & $\begin{array}{l}-\mathrm{Cl},-\mathrm{OH} \\
-\mathrm{COOH}\end{array}$ & $\begin{array}{l}\text { Hydrogen } \\
\text { bonding }\end{array}$ & RSDC & $\begin{array}{l}\text { [BMIM][Cl], } \\
130^{\circ} \mathrm{C}, 4 \mathrm{~h}\end{array}$ & - & - & TRS (73.2) & $\begin{array}{l}\text { Kept stable after } \\
\text { recycling five times }\end{array}$ & $\begin{array}{l}\text { Hu et al. } \\
(2016)\end{array}$ \\
\hline 16 & $\mathrm{SA}-\mathrm{SO}_{3} \mathrm{H}$ & $-\mathrm{Cl}$ & $\begin{array}{l}\text { Hydrogen } \\
\text { bonding }\end{array}$ & Ball-milled Avicel & $\begin{array}{c}\mathrm{H}_{2} \mathrm{O} \\
180^{\circ} \mathrm{C}, 12 \mathrm{~h}\end{array}$ & - & 5.7 & LA (52.2) & $\begin{array}{l}\text { Decreased by } 11 \% \\
\text { after five times }\end{array}$ & $\begin{array}{l}\text { Shen } \\
\text { et al. } \\
(2017)\end{array}$ \\
\hline 17 & CCSA & $\begin{array}{l}-\mathrm{Cl},-\mathrm{OH} \\
-\mathrm{COOH}\end{array}$ & $\begin{array}{l}\text { Hydrogen } \\
\text { bonding }\end{array}$ & Cellobiose & $\begin{array}{c}\mathrm{H}_{2} \mathrm{O} \\
120^{\circ} \mathrm{C}, 6 \mathrm{~h}\end{array}$ & - & 44.76 & - & $\begin{array}{l}\text { Leached about } \\
10-25 \% \text { of }-\mathrm{Cl} \text { after } \\
\text { recycling three } \\
\text { times }\end{array}$ & $\begin{array}{l}\text { Shen } \\
\text { et al. } \\
(2014)\end{array}$ \\
\hline 18 & $\begin{array}{l}\text { MCMPS-Cl- } \\
\mathrm{SO}_{3} \mathrm{H}\end{array}$ & $-\mathrm{Cl},-\mathrm{OH}$ & $\begin{array}{l}\text { Hydrogen } \\
\text { bonding }\end{array}$ & Pretreated MCC & $\begin{array}{l}{[\mathrm{BMIM}][\mathrm{Cl}]} \\
120^{\circ} \mathrm{C}, 6 \mathrm{~h}\end{array}$ & - & - & TRS (84.7) & $\begin{array}{l}\text { Kept stable after } \\
\text { recycling three } \\
\text { times }\end{array}$ & $\begin{array}{l}\text { Ding } \\
\text { et al. } \\
\text { (2019) }\end{array}$ \\
\hline 19 & BCSA-IL-Cu & IL groups & $\begin{array}{l}\text { Hydrogen } \\
\text { bonding }\end{array}$ & $\begin{array}{l}\text { Bamboo } \\
\text { pretreated by } \\
\text { microwave }\end{array}$ & $\begin{array}{l}\text { [BMIM][Cl]: } \\
\mathrm{H}_{2} \mathrm{O}=1: 1 \\
120^{\circ} \mathrm{C}, 2 \mathrm{~h}\end{array}$ & - & - & $\begin{array}{l}\text { TRS (27.6) } \\
\operatorname{HMF}(8.2)\end{array}$ & $\begin{array}{l}\text { Lost activity after } \\
\text { four runs }\end{array}$ & $\begin{array}{l}\text { Zhang } \\
\text { et al. } \\
\text { (2013) }\end{array}$ \\
\hline 20 & $\begin{array}{l}\text { Porous polymers } \\
\text { solid acids }\end{array}$ & $\begin{array}{l}\text { Boronic } \\
\text { acids } \\
\text { groups }\end{array}$ & $\begin{array}{l}\text { Reversible } \\
\text { chemical } \\
\text { bonding }\end{array}$ & Avicel & $\begin{array}{c}\mathrm{H}_{2} \mathrm{O} \\
120^{\circ} \mathrm{C}, 48 \mathrm{~h}\end{array}$ & - & 94.6 & - & - & $\begin{array}{l}\text { Yang } \\
\text { et al. } \\
\text { (2016) }\end{array}$ \\
\hline
\end{tabular}

GO, graphene oxide; PPSAs, porous polymeric solid acids; $\mathrm{CP}_{-} \mathrm{SO}_{3} \mathrm{H}$, sulfonated chloromethyl polystyrene resin; PTA, Phosphotungstic acid; MIL-101, a metal organic framework; LA, levulinic acid; SUCRA-SO ${ }_{3} \mathrm{H}$, sucralose-derived solid acid catalyst; $\mathrm{HA}-\mathrm{CC}-\mathrm{SO}_{3} \mathrm{H}$, hydrochloric acid-treated cellulose derived carbon solid acid catalyst; $\mathrm{SA}$, sucralose; $\mathrm{TsOH}$, p-toluenesulfonic acid; TRS, total reducing sugar, consisting of soluble glucose and oligosaccharides; MCMB, magnetic mesocarbon microbead; MCC, microcrystalline cellulose; CMC, chlorine-doped magnetic amorphous carbon; $\mathrm{RSDC}$, rice straw-derived cellulose; CCSA, a chlorine functionalized carbon-based solid acids; $M C M P S-C l-S O_{3} H$, a magnetically cellulasemimetic resin catalyst; BCSA, biochar sulfonic acid; HMF, 5-hydroxymethyl furfural; IL, 1-(trimethoxypropylsilane)-3-methyl imidazolium chloride. 


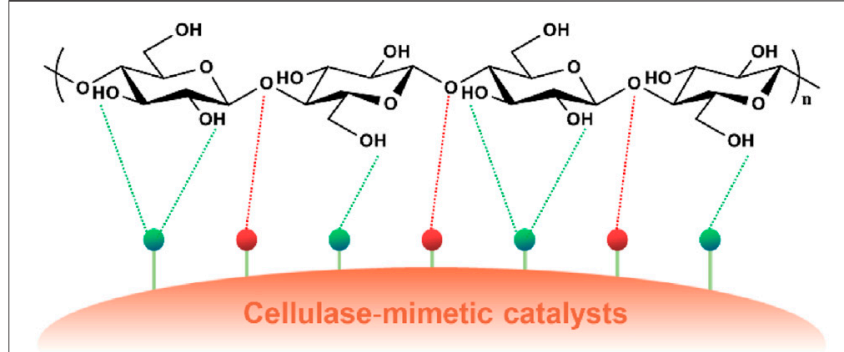

Binding sites (Phenolic-OH, $-\mathrm{COOH},-\mathrm{Cl},-\mathrm{Br},-\mathrm{NH}_{2},-\mathrm{NO}_{2}$, etc. )

Catalytic sites $\left(\mathrm{SO}_{3} \mathrm{H}, \mathrm{PTA}\right.$, etc. )

FIGURE 3 |A summary of functional groups as binding sites of cellulasemimetic solid acid catalysts and the proposed catalysis mechanism for cellulose hydrolysis.

cellulose-binding groups. The apparent activation energies for the hydrolysis of cellobiose and microcrystalline cellulose (Avicel) catalyzed by a polymer-based catalyst bearing chlorine $(-\mathrm{Cl})$ and $-\mathrm{SO}_{3} \mathrm{H}\left(\mathrm{CP}-\mathrm{SO}_{3} \mathrm{H}\right)$ were reduced to 78 and $83 \mathrm{~kJ} \mathrm{~mol}^{-1}$, respectively, which are much lower than those of sulfuric acidcatalyzed hydrolysis (133 and $170 \mathrm{~kJ} \mathrm{~mol}^{-1}$, respectively) (Figure 3; Shuai et al., 2012). The $\mathrm{CP}-\mathrm{SO}_{3} \mathrm{H}$ catalyst adsorbed both cellobiose and glucose and meanwhile showed higher affinity to cellobiose, which is presumably because more hydrogen bonds formed between the catalyst and cellobiose due to more hydroxyl groups available in cellobiose. The catalysts bearing $-\mathrm{NH}_{2}$ and $-\mathrm{OH}$ groups showed lower activity than that bearing $-\mathrm{Cl}$ but higher catalytic activity than support (Amberlyst-15) without any binding and catalytic sites (Shuai et al., 2012). This comparison indicates that a more electronegative group has higher affinity to cellulosic materials. Similarly, a metal-organic framework material (MIL-101) carrier bearing various electronegative groups $\left(\mathrm{X}=-\mathrm{Br},-\mathrm{NH}_{2},-\mathrm{Cl}\right.$, and $-\mathrm{NO}_{2}$ ) and phosphotungstic acids (PTA) (PTA@MIL-101-X) also showed enhanced catalyst-cellulose interactions (Figure 3). $\mathrm{NO}_{2}$ grafted catalyst PTA@MIL-101- $\mathrm{NO}_{2}$ achieved the highest glucose yield due to its highest affinity to cellulosic materials (Han et al., 2019). The efficiency of cellulose hydrolysis increases with the increase of the electronegativity $\left(-\mathrm{NO}_{2}>-\mathrm{Cl}>-\mathrm{NH}_{2}>-\mathrm{Br}\right)$ of the binding groups (Han et al., 2019), which is consistent with the observation by Shuai and Pan (Shuai et al., 2012). A series of solid acid catalysts bearing - $\mathrm{Cl}$ were synthesized with different methods and enhanced glucose yields by about 2.5 times (93\%) compared to the catalysts without - $\mathrm{Cl}$ (37\%) (Pang et al., 2014; Shen et al., 2014; Zhao et al., 2014; Zuo et al., 2014; Hu et al., 2016; Yang et al., 2016; Shen et al., 2017; Shen et al., 2018; Ding et al., 2019; Li et al., 2020; Li et al., 2020). These catalysts follow the same binding mechanism that proposed above for promoting cellulose hydrolysis efficiency.

Ionic liquids consisting of electronegative anions such as $-\mathrm{Cl}$ and $-\mathrm{Br}$ that can effectively disrupt cellulose crystalline via forming new hydrogen bonds with cellulose hydroxyls are also potential candidates for cellulose-binding sites. A sulfonated biochar material grafted with ionic liquid

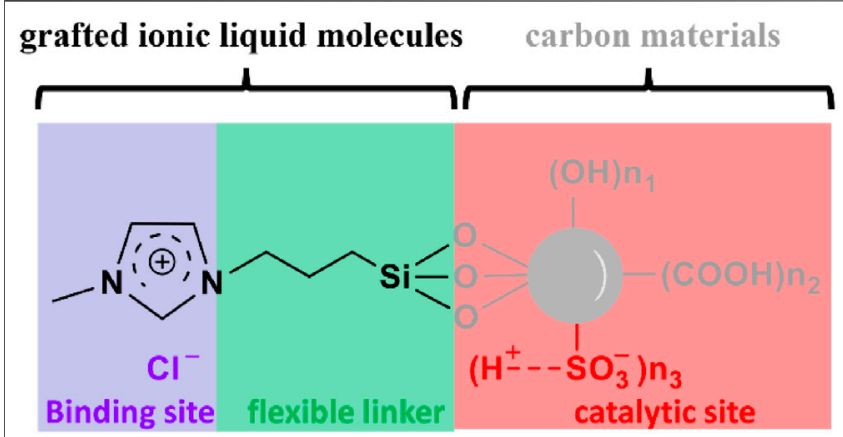

FIGURE 4 | A sulfonated biochar material grafted with ionic liquid molecules as a cellulase-mimetic solid acid catalyst.

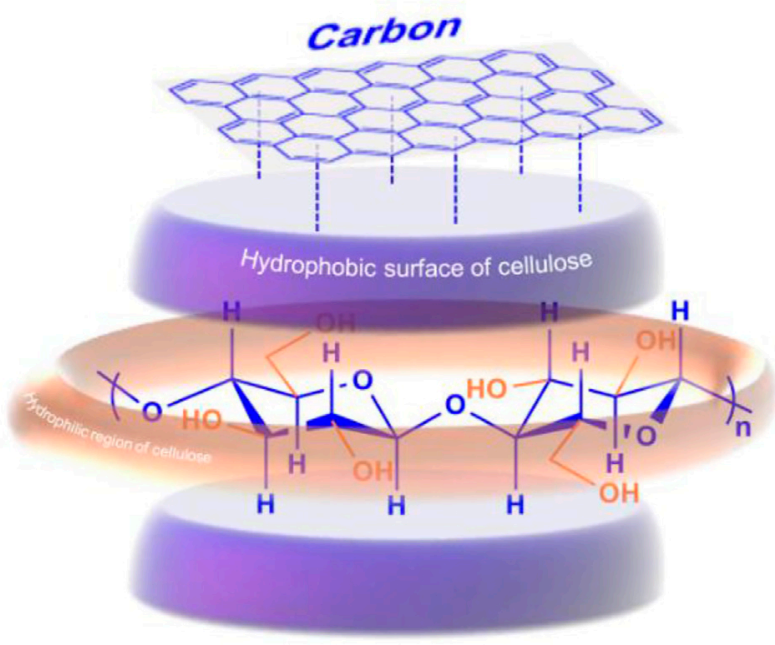

FIGURE 5 | Adsorption of cellulose onto carbon materials via the hydrophobic interactions between the axial faces of cellulosic materials and the polycyclic aromatic rings of carbon materials.

molecules (e.g. 1-(trimethoxy propyl silane)-3methylimidazolium chloride) could convert about $35.8 \%$ of microwave-pretreated bamboo $(750 \mathrm{~W})$ at $120^{\circ} \mathrm{C}$ in $2 \mathrm{~h}$ (Figure 4; Zhang et al., 2013). The IL groups flexibly joined to biochar, similar to the $\mathrm{CBD}$ and interdomain linker of cellulase, can efficiently break the hydrogen bonding network of cellulose crystalline and form new hydrogen bonds with cellulose hydroxyls (Figure 4). Therefore, ionic liquid molecules, compared to other binding sites discussed previously, can act as very promising binding sites for converting cellulose and real lignocellulosic biomass that contain crystalline structures. Such a unique property of ionic liquids is worth more attention in future work towards developing a more practical catalyst for biorefineries.

\section{(B) Binding Via Hydrophobic Interaction}

Inspired by the hydrophobic interaction mechanism between cellulose and the CBD of cellulase, Mosier et al. screened amino 


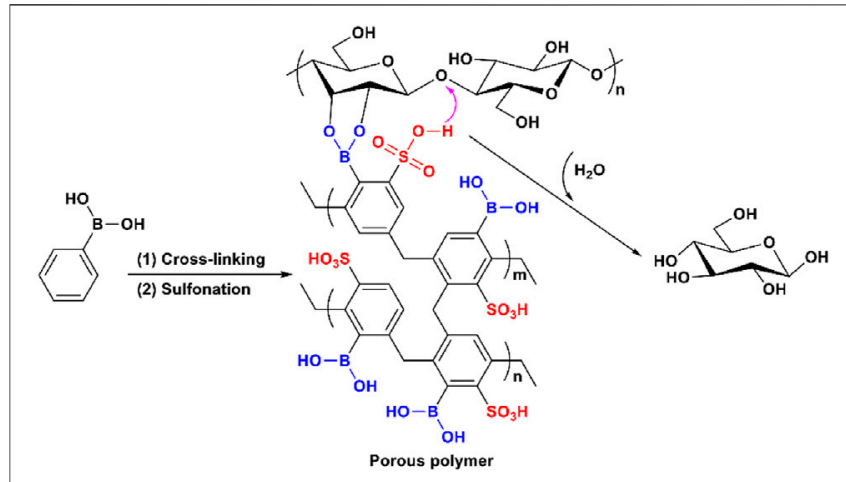

FIGURE 6 | Proposed cellulose hydrolysis mechanism for the cellulasemimetic catalyst bearing boronic and sulfonic acids on porous polymer.

acids that could bind cellulose. Indoles and tryptophan blue that have aromatic moieties were found to have high affinities to cellulose (Mosier et al., 2004), which highly indicates that hydrophobic interactions are essential for cellulose-cellulase binding other than hydrogen bonding interactions. Consistently, a variety of carbon materials demonstrate high adsorption capacity for cellulose via hydrophobic interactions (Chung et al., 2012; Chung et al., 2015; Onda et al., 2008; Yabushita et al., 2016). For example, a mesoporous carbon material with a pore diameter of $3.2 \mathrm{~nm}$ was able to adsorb soluble sugars (glucose and cello-oligosaccharides) in a quantity up to $670 \mathrm{mg} \mathrm{g}^{-1}$ (Chung et al., 2012); $1 \mathrm{~g}$ of a zeolite-templated carbon material (ZTC) could adsorb $800 \mathrm{mg}$ of cellulosic molecules (Chung et al., 2015). The adsorption is mainly attributed to the $\mathrm{CH}-\pi$ hydrophobic interactions between the $\mathrm{CH}$ groups on the axial face of cellulose and the polycyclic aromatics of carbon materials, whereas no hydrogen bonding interaction between the mesoporous carbon materials and cellulosic materials was observed (Figure 5; Chung et al., 2012). The adsorption strength increased with the increasing number of glucose units (Chung et al., 2015), which is consistent with the study reported by Shuai and Pan (Shuai et al., 2012). Negative enthalpy change was observed during the adsorption of cello-oligosaccharides on carbon materials due to the $\mathrm{CH}-\pi$ interactions (Yabushita et al., 2014). The hydrophobic interaction between cellulose and carbon aromatic rings exists considerably only in the presence of water as a solvent because water molecules can drive the interaction of hydrophobic interfaces of cellulose and carbon materials (Meyer et al., 2006). The GO sheets in the carbon-based solid acids can also guide the assembly of cellulose via both $\pi-\pi$ and hydrogen bonding interactions, facilitating the spread of glucose and reducing the occurrence of side reactions (Zhang et al., 2017).

\section{(C) Binding Via Reversible Chemical Bonding}

Other than the aforementioned physical interactions, a reversible chemical binding strategy was also explored by
Yang and Pan (Figure 6; Yang et al., 2016). Boronic acid can form reversible covalent bonds with the vicinal hydroxyls of carbohydrates (Springsteen et al., 2002; John Griffin et al., 2004; Matsumoto et al., 2005) and therefore can be used as the binding site of cellulase-mimetic solid acids. A bifunctional porous polymer bearing boronic and sulfonic acids achieved glucose yields of $43 \%$ in $24 \mathrm{~h}$ and $95 \%$ in $48 \mathrm{~h}$, respectively, for cellulose hydrolysis (Figure 5). Specifically, the boronic acid group reacts with two vicinal hydroxyl groups of cellulose to form a reversible five-element ring structure, which brings cellulose close to the catalytic sites of the catalyst for hydrolysis.

\section{PERSPECTIVES}

Cellulase-mimetic solid acid catalysts are excellent candidates to replace traditional solid acid catalysts, in which the catalytic sites and binding sites play a crucial role in efficient cellulose hydrolysis. Cellulase-mimetic solid acids usually demonstrate higher hydrolysis efficiencies than conventional solid acids without tailored catalytic and binding sites; however, most of the current studies use cellobiose, amorphous cellulose, or ball-milled cellulose as the substrate for catalyst performance testing. The selective hydrolysis of more practical substrates such as untreated cellulose that has crystalline structures and lignocellulosic biomass that has unremoved lignin is still challenging and problematic for such a type of solid catalysts due to the limited interaction between the solid catalysts and bulk cellulosic materials. A few attempts to use pretreated lignocelluloses as substrates only got limited glucose yields (almost all less than 20\%, Table 1) (Zhang et al., 2012; Hu et al., 2014; Hu et al., 2016). To overcome the limitation of current catalysts for hydrolyzing crystalline cellulose and lignocellulosic materials, future research could be focused on green solvents or activators that can be developed as a cellulase-mimetic morphogenesis for disrupting the crystalline structure of cellulose prior to the binding (Brandt et al., 2013; da Costa Lopes et al., 2013; Lee et al., 2014).

Developing water-soluble cellulase-mimetic solid acid catalysts could be a potential solution to the slow mass transfer problem. The water solubility of such catalysts could facilitate the fast adsorption of water-soluble catalyst molecules to cellulose surface. This is particularly necessary for hydrolyzing cellulosic materials that exist in the solid form and with lignin. In this way, the water-soluble catalyst can be easily separated from lignin. With molecular design, temperature-, $\mathrm{pH}$ - and light-sensitive functional groups can be incorporated into the water-soluble catalysts to manipulate their solubility and absorptivity for the recovery of the catalysts after hydrolysis. The effects of lignin and hemicellulose on stability of cellulase-mimetic acid catalysts remains to be further studied.

The structure of the polyaromatic ring as a binding site of cellulase-mimetic catalysts has a great application prospect in future work. Although carbon materials have promising cellulose adsorption potential, the unique structural features 
of carbon polyaromatics are not fully optimized. Typically, the activity is defined by aromatic structure (Basal plane) and the specific functional groups on edges while other important properties of carbon such as electron density, hybridizations, defects, and electrophilicity are not well understood. The current advancement in material development may provide the internal local surface constrains for better interaction between the catalyst framework and cellulose in an intracrystalline free space for enhanced enzyme-like activity. Furthermore, a deeper understanding of the nature of the active and reactive sites could provide a pathway towards next-generation functionalized polyaromatic as a biomimetic catalyst for cellulose hydrolysis.

\section{REFERENCES}

Asensio, J. L., Ardá, A., Cañada, F. J., and Jiménez-Barbero, J. (2012). Carbohydrate-aromatic Interactions. Acc. Chem. Res. 46, 946-954. doi:10.1021/ar300024d

Badieyan, S., Bevan, D. R., and Zhang, C. (2012). Probing the Active Site Chemistry of $\beta$-Glucosidases Along the Hydrolysis Reaction Pathway. Biochemistry 51, 8907-8918. doi:10.1021/bi300675x

Berlin, A., Maximenko, V., Gilkes, N., and Saddler, J. (2007). Optimization of Enzyme Complexes for Lignocellulose Hydrolysis. Biotechnol. Bioeng. 97, 287-296. doi:10.1002/bit.21238

Brandt, A., Gräsvik, J., Hallett, J. P., and Welton, T. (2013). Deconstruction of Lignocellulosic Biomass with Ionic Liquids. Green. Chem. 15, 550-583. doi:10.1039/C2GC36364J

Brown, R., and Jurasek, L. (1979). Hydrolysis of Cellulose: Mechanisms of Enzymatic and Acid Catalysis. Carbohydr. Res. 181. doi:10.1021/ba-1979-0181

Chen, Z., Li, Q., Xiao, Y., Zhang, C., Fu, Z., Liu, Y., et al. (2019). Acid-base Synergistic Catalysis of Biochar Sulfonic Acid Bearing Polyamide for Microwave-Assisted Hydrolysis of Cellulose in Water. Cellulose 26, 751-762. doi:10.1007/s10570-018-2098-3

Cho, E. J., Lee, S. J., Lee, K., Lee, D.-S., Lee, Y. J., and Bae, H.-J. (2015). A Reusable Biomimetic Magnetic Nanoenzyme for Cellulosic Biomass Degradation. Bioenerg. Res. 8, 788-795. doi:10.1007/s12155-014-9559-9

Cho, E. J., Song, Y., Lee, Y. J., and Bae, H.-J. (2016). Preparation and Characterization of Novel Green Magnetic Nanocatalyst for Cellulosic Biomass Degradation Under Mild Conditions. J. Ind. Eng. Chem. 40, 185-190. doi:10.1016/j.jiec.2016.06.022

Chung, P.-W., Charmot, A., Gazit, O. M., and Katz, A. (2012). Glucan Adsorption on Mesoporous Carbon Nanoparticles: Effect of Chain Length and Internal Surface. Langmuir 28, 15222-15232. doi:10.1021/la3030364

Chung, P.-W., Yabushita, M., To, A. T., Bae, Y., Jankolovits, J., Kobayashi, H., et al. (2015). Long-chain Glucan Adsorption and Depolymerization in ZeoliteTemplated Carbon Catalysts. ACS Catal. 5, 6422-6425. doi:10.1021/ acscatal.5b01172

da Costa Lopes, A. M., João, K. G., Morais, A. R. C., Bogel-Łukasik, E., and BogelŁukasik, R. (2013). Ionic Liquids as a Tool for Lignocellulosic Biomass Fractionation. Sustain. Chem. Process 1, 1-31. doi:10.1186/2043-7129-1-3

Ding, X., Guo, Y., Liu, S., Gong, Z., Dai, X., Li, N., et al. (2019). High-efficiency Depolymerization of Microcrystalline Cellulose in 1-Butyl-3-Methylimidazolium Chloride Over a Magnetically Recoverable Cellulase-Mimetic Resin Catalyst. j biobased mat bioenergy 13, 389-394. doi:10.1166/jbmb.2019.1862

dos Santos, A. C., Ximenes, E., Kim, Y., and Ladisch, M. R. (2019). Lignin-Enzyme Interactions in the Hydrolysis of Lignocellulosic Biomass. Trends Biotechnol. 37, 518-531. doi:10.1016/j.tibtech.2018.10.010

Gan, L., Zhu, J., and Lv, L. (2017). Cellulose Hydrolysis Catalyzed by Highly Acidic Lignin-Derived Carbonaceous Catalyst Synthesized via Hydrothermal Carbonization. Cellulose 24, 5327-5339. doi:10.1007/s10570-017-1515-3

\section{AUTHOR CONTRIBUTIONS}

GX and XL developed the concept and wrote the manuscript. LS modified the concept and proofread the manuscript for language and technicalities.

\section{FUNDING}

This work was supported by National Natural Science Foundation of China (No. 32071716, 31870559, and 31901262), Outstanding Youth Funding of National Forestry and Grassland Administration (20201326005), Outstanding Youth Funding (xjq201923) of Fujian Agriculture and Forestry University.

Georgelis, N., Yennawar, N. H., and Cosgrove, D. J. (2012). Structural Basis for Entropy-Driven Cellulose Binding by a Type-A Cellulose-Binding Module (CBM) and Bacterial Expansin. Proc. Natl. Acad. Sci. 109, 14830-14835. doi:10.1073/pnas.1213200109

Han, J., Wang, Y., Wan, J., and Ma, Y. (2019). Catalytic Hydrolysis of Cellulose by Phosphotungstic Acid-Supported Functionalized Metal-Organic Frameworks with Different Electronegative Groups. Environ. Sci. Pollut. Res. 26, 15345-15353. doi:10.1007/s11356-019-04923-7

Hara, M. (2010). Biomass Conversion by a Solid Acid Catalyst. Energy Environ. Sci. 3, 601-607. doi:10.1039/B922917E

Hu, L., Li, Z., Wu, Z., Lin, L., and Zhou, S. (2016). Catalytic Hydrolysis of Microcrystalline and rice Straw-Derived Cellulose Over a Chlorine-Doped Magnetic Carbonaceous Solid Acid. Ind. Crops Prod. 84, 408-417. doi:10.1016/j.indcrop.2016.02.039

Hu, L., Lin, L., Wu, Z., Zhou, S., and Liu, S. (2015). Chemocatalytic Hydrolysis of Cellulose into Glucose Over Solid Acid Catalysts. Appl. Catal. B: Environ. 174175, 225-243. doi:10.1016/j.apcatb.2015.03.003

Hu, S., Jiang, F., and Hsieh, Y.-L. (2015). 1D Lignin-Based Solid Acid Catalysts for Cellulose Hydrolysis to Glucose and Nanocellulose. ACS Sust. Chem. Eng. 3, 2566-2574. doi:10.1021/acssuschemeng.5b00780

Hu, S., Smith, T. J., Lou, W., and Zong, M. (2014). Efficient Hydrolysis of Cellulose Over a Novel Sucralose-Derived Solid Acid with Cellulose-Binding and Catalytic Sites. J. Agric. Food Chem. 62, 1905-1911. doi:10.1021/jf405712b

Huang, A. A. (1975). Enzymatic Hydrolysis of Cellulose to Sugar. Biotechnol. Bioeng. Symp. 5, 245-252. doi:10.1007/978-1-4615-9290-7-84

Huang, Y.-B., and Fu, Y. (2013). Hydrolysis of Cellulose to Glucose by Solid Acid Catalysts. Green. Chem. 15, 1095-1111. doi:10.1039/c3gc40136g

John Griffin, G., and Shu, L. (2004). Solvent Extraction and Purification of Sugars from Hemicellulose Hydrolysates Using Boronic Acid Carriers. J. Chem. Technol. Biotechnol. 79, 505-511. doi:10.1002/jctb.1013

Kitano, M., Yamaguchi, D., Suganuma, S., Nakajima, K., Kato, H., Hayashi, S., et al. (2009). Adsorption-Enhanced Hydrolysis of $\beta-1,4-G l u c a n$ on Graphene-Based Amorphous Carbon Bearing $\mathrm{SO} 3 \mathrm{H}, \mathrm{COOH}$, and $\mathrm{OH}$ Groups. Langmuir 25, 5068-5075. doi:10.1021/la8040506

Lee, H. V., Hamid, S. B. A., and Zain, S. K. (2014). Conversion of Lignocellulosic Biomass to Nanocellulose: Structure and Chemical Process. Scientific World J. 2014, 1-20. doi:10.1155/2014/631013

Li, H.-X., Zhang, X., Wang, Q., Yang, D., Cao, Q., and Jin, L. e. (2020). Study on the Hydrolysis of Cellulose with the Regenerable and Recyclable Multifunctional Solid Acid as a Catalyst and its Catalytic Hydrolytic Kinetics. Cellulose 27, 285-300. doi:10.1007/s10570-019-02777-3

Li, H. X., Shi, W. J., Zhang, X., Liu, P., Cao, Q., and Jin, L. e. (2020). Catalytic Hydrolysis of Cellulose to Total Reducing Sugars with Superior Recyclable Magnetic Multifunctional MCMB-based Solid Acid as a Catalyst. J. Chem. Technol. Biotechnol. 95, 770-780. doi:10.1002/jctb.6262

Matsumoto, M., Ueba, K., and Kondo, K. (2005). Separation of Sugar by Solvent Extraction with Phenylboronic Acid and Trioctylmethylammonium Chloride. Separat. Purif. Techn. 43, 269-274. doi:10.1016/j.seppur.2004.11.010 
Meyer, E. E., Rosenberg, K. J., and Israelachvili, J. (2006). Recent Progress in Understanding Hydrophobic Interactions. Proc. Natl. Acad. Sci. 103, 15739-15746. doi:10.1073/pnas.0606422103

Mission, E. G., Quitain, A. T., Sasaki, M., and Kida, T. (2017). Synergizing Graphene Oxide with Microwave Irradiation for Efficient Cellulose Depolymerization into Glucose. Green. Chem. 19, 3831-3843. doi:10.1039/ C7GC01691C

Mosier, N. S., Wilker, J. J., and Ladisch, M. R. (2004). Rapid Chromatography for Evaluating Adsorption Characteristics of Cellulase Binding Domain Mimetics. Biotechnol. Bioeng. 86, 756-764. doi:10.1002/bit.20104

Onda, A., Ochi, T., and Yanagisawa, K. (2008). Selective Hydrolysis of Cellulose into Glucose Over Solid Acid Catalysts. Green. Chem. 10, 1033-1037. doi:10.1039/B808471H

Orozco, A. M., Al-Muhtaseb, A. a. H., Albadarin, A. B., Rooney, D., Walker, G. M., and Ahmad, M. N. M. (2011). Acid-catalyzed Hydrolysis of Cellulose and Cellulosic Waste Using a Microwave Reactor System. RSC Adv. 1, 839-846. doi:10.1039/C1RA00329A

Palkovits, R., Tajvidi, K., Procelewska, J., Rinaldi, R., and Ruppert, A. (2010). Hydrogenolysis of Cellulose Combining Mineral Acids and Hydrogenation Catalysts. Green. Chem. 12, 972-978. doi:10.1039/ C000075B

Pang, J., Wang, A., Zheng, M., and Zhang, T. (2010). Hydrolysis of Cellulose into Glucose Over Carbons Sulfonated at Elevated Temperatures. Chem. Commun. 46, 6935-6947. doi:10.1039/c0cc02014a

Pang, Q., Wang, L., Yang, H., Jia, L., Pan, X., and Qiu, C. (2014). Cellulose-derived Carbon Bearing - $\mathrm{Cl}$ and -SO3H Groups as a Highly Selective Catalyst for the Hydrolysis of Cellulose to Glucose. RSC Adv. 4, 41212-41218. doi:10.1039/ C4RA05520A

Qiao, Y., Teng, N., Zhai, C., Na, H., and Zhu, J. (2018). High Efficient Hydrolysis of Cellulose into Sugar by Chemical Catalytic Method. Prog. Chem. 30, 1415-1423. doi:10.7536/PC180126

Rabinovich, M. L., Melnick, M. S., and Bolobova, A. V. (2002). The Structure and Mechanism of Action of Cellulolytic Enzymes. Biochemistry 67, 850-871. doi:10.1023/a:1019958419032

Rinaldi, R., and Schüth, F. (2010). Acid Hydrolysis of Cellulose as the Entry point into Biorefinery Schemes. ChemSusChem 3, 296. doi:10.1002/cssc.201090010

Shen, F., Guo, T., Bai, C., Qiu, M., and Qi, X. (2018). Hydrolysis of Cellulose with One-Pot Synthesized Sulfonated Carbonaceous Solid Acid. Fuel Process. Techn. 169, 244-247. doi:10.1016/j.fuproc.2017.10.015

Shen, F., Smith, R. L., Li, L., Yan, L., and Qi, X. (2017). Eco-friendly Method for Efficient Conversion of Cellulose into Levulinic Acid in Pure Water with Cellulase-Mimetic Solid Acid Catalyst. ACS Sust. Chem. Eng. 5, 2421-2427. doi:10.1021/acssuschemeng.6b02765

Shen, S., Cai, B., Wang, C., Li, H., Dai, G., and Qin, H. (2014). Preparation of a Novel Carbon-Based Solid Acid from Cocarbonized Starch and Polyvinyl Chloride for Cellulose Hydrolysis. Appl. Catal. A: Gen. 473, 70-74. doi:10.1016/j.apcata.2013.12.037

Shimizu, K.-i., and Satsuma, A. (2011). Toward a Rational Control of Solid Acid Catalysis for Green Synthesis and Biomass Conversion. Energ. Environ. Sci. 4, 3140-3153. doi:10.1039/C1EE01458G

Shuai, L., and Pan, X. (2012). Hydrolysis of Cellulose by Cellulase-Mimetic Solid Catalyst. Energ. Environ. Sci. 5, 6889-6894. doi:10.1039/ c2ee03373a

Springsteen, G., and Wang, B. (2002). A Detailed Examination of Boronic AcidDiol Complexation. Tetrahedron 58, 5291-5300. doi:10.1016/S0040-4020(02) 00489-1

Sugano, Y., Vestergaard, M. d. C., Saito, M., and Tamiya, E. (2011). A Carbon Nanotube Structured Biomimetic Catalyst for Polysaccharide Degradation. Chem. Commun. 47, 7176-7188. doi:10.1039/c1cc10927h

Suganuma, S., Nakajima, K., Kitano, M., Yamaguchi, D., Kato, H., Hayashi, S., et al. (2008). Hydrolysis of Cellulose by Amorphous Carbon Bearing SO3H, $\mathrm{COOH}$, and OH Groups. J. Am. Chem. Soc. 130, 12787-12793. doi:10.1021/ja803983h

Toda, M., Takagaki, A., Okamura, M., Kondo, J. N., Hayashi, S., Domen, K., et al. (2005). Biodiesel Made with Sugar Catalyst. Nature 438, 178. doi:10.1038/ 438178a

Van de Vyver, S., Peng, L., Geboers, J., Schepers, H., de Clippel, F., Gommes, C. J., et al. (2010). Sulfonated Silica/carbon Nanocomposites as Novel Catalysts for Hydrolysis of Cellulose to Glucose. Green. Chem. 12, 1560-1563. doi:10.1039/c0gc00235f
Verma, D., Tiwari, R., and Sinha, A. K. (2013). Depolymerization of Cellulosic Feedstocks Using Magnetically Separable Functionalized Graphene Oxide. RSC Adv. 3, 13265-13272. doi:10.1039/C3RA41025K

Wang, J., Xi, J., and Wang, Y. (2015). Recent Advances in the Catalytic Production of Glucose from Lignocellulosic Biomass. Green. Chem. 17, 737-751. doi:10.1039/c4gc02034k

Wang, S., Sima, G., Cui, Y., Chang, L., and Gan, L. (2020). Efficient Hydrolysis of Cellulose to Glucose Catalyzed by Lignin-Derived Mesoporous Carbon Solid Acid in Water. Chin. J. Chem. Eng. 28, 1866-1874. doi:10.1016/j.cjche.2020.03.012

Yabushita, M., Kobayashi, H., and Fukuoka, A. (2014). Catalytic Transformation of Cellulose into Platform Chemicals. Appl. Catal. B: Environ. 145, 1-9. doi:10.1016/j.apcatb.2013.01.052

Yabushita, M., Kobayashi, H., Hasegawa, J.-y., Hara, K., and Fukuoka, A. (2014). Entropically Favored Adsorption of Cellulosic Molecules onto Carbon Materials through Hydrophobic Functionalities. ChemSusChem 7, 1443-1450. doi:10.1002/cssc.201301296

Yabushita, M. (2016). "Mechanistic Study of Cellulose Hydrolysis by Carbon Catalysts," in A Study on Catalytic Conversion of Non-food Biomass Intochemicals: Fusion of Chemical Sciences and Engineering. Editor M. Yabushita (Singapore: Springer Singapore), 77-112. doi:10.1007/978-981-10-0332-5_3

Yabushita, M., Techikawara, K., Kobayashi, H., Fukuoka, A., and Katz, A. (2016). Zeolite-templated Carbon Catalysts for Adsorption and Hydrolysis of Cellulose-Derived Long-Chain Glucans: Effect of Post-synthetic Surface Functionalization. ACS Sust. Chem. Eng. 4, 6844-6851. doi:10.1021/ acssuschemeng.6b01796

Yang, Q., and Pan, X. (2016). Bifunctional Porous Polymers Bearing Boronic and Sulfonic Acids for Hydrolysis of Cellulose. ACS Sust. Chem. Eng. 4, 4824-4830. doi:10.1021/acssuschemeng.6b01102

Yang, Q., and Pan, X. (2021). Introducing Hydroxyl Groups as CelluloseBinding Sites into Polymeric Solid Acids to Improve Their Catalytic Performance in Hydrolyzing Cellulose. Carbohydr. Polym. 261, 117895. doi:10.1016/j.carbpol.2021.117895

Yang, Q., and Pan, X. (2016). Synthesis and Application of Bifunctional Porous Polymers Bearing Chloride and Sulfonic Acid as Cellulase-Mimetic Solid Acids for Cellulose Hydrolysis. Bioenerg. Res. 9, 578-586. doi:10.1007/s12155-015-9702-2

Yu, F., Smet, M., Dehaen, W., and Sels, B. F. (2016). Water-soluble Sulfonated Hyperbranched Poly(arylene Oxindole) Catalysts as Functional Biomimics of Cellulases. Chem. Commun. 52, 2756-2759. doi:10.1039/C5CC08742B

Zechel, D. L., and Withers, S. G. (2000). Glycosidase Mechanisms: Anatomy of a Finely Tuned Catalyst. Acc. Chem. Res. 33, 11-18. doi:10.1021/ $\operatorname{ar} 970172+$

Zhang, C., Fu, Z., Dai, B., Zen, S., Liu, Y., Xu, Q., et al. (2013). Chlorocuprate Ionic Liquid Functionalized Biochar Sulfonic Acid as an Efficiently Biomimetic Catalyst for Direct Hydrolysis of Bamboo Under Microwave Irradiation. Ind. Eng. Chem. Res. 52, 11537-11543. doi:10.1021/ie401100x

Zhang, C., Fu, Z., Liu, Y. C., Dai, B., Zou, Y., Gong, X., et al. (2012). Ionic LiquidFunctionalized Biochar Sulfonic Acid as a Biomimetic Catalyst for Hydrolysis of Cellulose and Bamboo Under Microwave Irradiation. Green. Chem. 14, 1928-1935. doi:10.1039/c2gc35071h

Zhang, C., Wang, H., Liu, F., Wang, L., and He, H. (2013). Magnetic Core-Shell Fe3O4@C-So3h Nanoparticle Catalyst for Hydrolysis of Cellulose. Cellulose 20, 127-134. doi:10.1007/s10570-012-9839-5

Zhang, M., Wu, M., Liu, Q., Wang, X., Lv, T., and Jia, L. (2017). Graphene Oxide Mediated Cellulose-Derived Carbon as a Highly Selective Catalyst for the Hydrolysis of Cellulose to Glucose. Appl. Catal. A: Gen. 543, 218-224. doi:10.1016/j.apcata.2017.06.033

Zhao, X., Wang, J., Chen, C., Huang, Y., Wang, A., and Zhang, T. (2014). Graphene Oxide for Cellulose Hydrolysis: How it Works as a Highly Active Catalyst. Chem. Commun. 50, 3439-3442. doi:10.1039/ c3cc49634a

Zhou, Z., Liu, D., and Zhao, X. (2021). Conversion of Lignocellulose to Biofuels and Chemicals via Sugar Platform: An Updated Review on Chemistry and Mechanisms of Acid Hydrolysis of Lignocellulose. Renew. Sust. Energ. Rev. 146, 111169. doi:10.1016/j.rser.2021.111169

Zhu, J., Gan, L., Li, B., and Yang, X. (2016). Synthesis and Characteristics of LigninDerived Solid Acid Catalysts for Microcrystalline Cellulose Hydrolysis. Korean J. Chem. Eng. 34, 110-117. doi:10.1007/s11814-016-0220-5 
Zhu, S., Xu, J., Cheng, Z., Kuang, Y., Wu, Q., Wang, B., et al. (2020). Catalytic Transformation of Cellulose into Short Rod-like Cellulose Nanofibers and Platform Chemicals Over Lignin-Based Solid Acid. Appl. Catal. B: Environ. 268, 118732-118744. doi:10.1016/ j.apcatb.2020.118732

Zuo, Y., Zhang, Y., and Fu, Y. (2014). Catalytic Conversion of Cellulose into Levulinic Acid by a Sulfonated Chloromethyl Polystyrene Solid Acid Catalyst. ChemCatChem 6, 753-757. doi:10.1002/cctc.201300956

Conflict of Interest: The authors declare that the research was conducted in the absence of any commercial or financial relationships that could be construed as a potential conflict of interest.
Publisher's Note: All claims expressed in this article are solely those of the authors and do not necessarily represent those of their affiliated organizations, or those of the publisher, the editors and the reviewers. Any product that may be evaluated in this article, or claim that may be made by its manufacturer, is not guaranteed or endorsed by the publisher.

Copyright $\odot 2021$ Yang, Luo and Shuai. This is an open-access article distributed under the terms of the Creative Commons Attribution License (CC BY). The use, distribution or reproduction in other forums is permitted, provided the original author(s) and the copyright owner(s) are credited and that the original publication in this journal is cited, in accordance with accepted academic practice. No use, distribution or reproduction is permitted which does not comply with these terms. 